\title{
Long non-coding RNA SNHG16 contributes to progression of carotid atherosclerosis by regulating miR-30c-5p/ADAM10 axis
}

\section{Type}

Research paper

\section{Keywords}

Carotid atherosclerosis, miR-30c-5p, Lnc-SNHG16, ADAM10

\begin{abstract}
Introduction

Carotid atherosclerosis (CAS) is one of the main causes of cerebral infarction in the ageing population. Long non-coding RNA small nucleolar RNA host gene 16 (Inc-SNHG16) could promote the development of atherosclerosis. However, the mechanism of Inc-SNHG16 in CAS remains vague.

\section{Material and methods}

The expression levels of Inc-SNHG16, microRNA-30c-5p (miR-30c-5p) and disintegrin and metalloproteinase 10 (ADAM10) were detected by real-time quantitative polymerase chain reaction (RT-qPCR). Cell viability and migration were detected by 3-(4, 5-dimethyl-2-thiazolyl)-2,

5-diphenyl-2-H-tetrazolium bromide (MTT) and transwell assays, severally. The levels of interleukin-6 (IL-6), IL- $\beta$ and tumor necrosis factor- $\alpha$ (TNF- $\alpha$ ) were assessed by enzyme-linked immunosorbent assay (ELISA). Protein levels of spinal muscular atrophy (SMA), calponin and ADAM10 were examined by western blot assay. The binding relationship between miR-30c-5p and Inc-SNHG16 or ADAM10 was predicted by Starbase, then verified by the dual-luciferase reporter assay.
\end{abstract}

\section{Results}

Lnc-SNHG16 and ADAM10 were increased, and miR-30c-5p was decreased in CAS patient and oxidized low-density lipoprotein (ox-LDL)-treated human aortic smooth muscle cells (hASMCs). LncSNHG16 silencing repressed cell viability, migration, inflammation, facilitated differentiation in ox-LDLtreated hASMCs. Moreover, mechanical analysis proved that Inc-SNHG16 improved ADAM10 expression by sponging $\mathrm{miR}-30 \mathrm{c}-5 \mathrm{p}$.

\section{Conclusions}

Our data indicated that Inc-SNHG16 could regulate the progression of ox-LDL induced CAS model by the miR-30c-5p/ADAM10 axis, implying a potential therapeutic strategy for CAS 
1 Long non-coding RNA SNHG16 contributes to progression of carotid atherosclerosis by regulating miR-30c-5p/ADAM10 axis

3

4

5 Running title: Role of lnc-SNHG16/miR-30c-5p/ADAM10 in carotid atherosclerosis

6

$7 \quad$ List of abbreviations

8 CAS: Carotid atherosclerosis

9 lnc-SNHG16: Long non-coding RNA small nucleolar RNA host gene 16

10 RT-qPCR: real-time quantitative polymerase chain reaction

11 SMA: spinal muscular atrophy

12 ox-LDL: oxidized low-density lipoprotein

13 hASMCs: human aortic smooth muscle cells

14

15 
ABSTRACT

2 Introduction: Carotid atherosclerosis (CAS) is one of the main causes of cerebral

3 infarction in the ageing population. Long non-coding RNA small nucleolar RNA host

4 gene 16 (lnc-SNHG16) could promote the development of atherosclerosis. However,

5 the mechanism of lnc-SNHG16 in CAS remains vague.

6 Material and Methods: The expression levels of lnc-SNHG16, microRNA-30c-5p

7 (miR-30c-5p) and disintegrin and metalloproteinase 10 (ADAM10) were detected by

8 real-time quantitative polymerase chain reaction (RT-qPCR). Cell viability and

9 migration were detected by 3-(4, 5-dimethyl-2-thiazolyl)-2, 5-diphenyl-2-H-

10 tetrazolium bromide (MTT) and transwell assays, severally. The levels of interleukin-

116 (IL-6), IL- $\beta$ and tumor necrosis factor- $\alpha$ (TNF- $\alpha$ ) were assessed by enzyme-linked

12 immunosorbent assay (ELISA). Protein levels of spinal muscular atrophy (SMA),

13 calponin and ADAM10 were examined by western blot assay. The binding relationship

14 between miR-30c-5p and lnc-SNHG16 or ADAM10 was predicted by Starbase, then

15 verified by the dual-luciferase reporter assay.

16 Results: Lnc-SNHG16 and ADAM10 were increased, and miR-30c-5p was decreased

17 in CAS patient and oxidized low-density lipoprotein (ox-LDL)-treated human aortic

18 smooth muscle cells (hASMCs). Lnc-SNHG16 silencing repressed cell viability,

19 migration, inflammation, facilitated differentiation in ox-LDL-treated hASMCs.

20 Moreover, mechanical analysis proved that lnc-SNHG16 improved ADAM10

21 expression by sponging miR-30c-5p.

22 Conclusion: Our data indicated that lnc-SNHG16 could regulate the progression of ox-

23 LDL induced CAS model by the miR-30c-5p/ADAM10 axis, implying a potential

24 therapeutic strategy for CAS

25 Key words: Lnc-SNHG16, miR-30c-5p, ADAM10, carotid atherosclerosis

\section{Introduction}

28 As a manifestation of systemic atherosclerosis in the carotid artery, carotid 29 atherosclerosis (CAS) has become a leading cause of cerebral infarction in the aging 30 population [1]. Currently, carotid intima-media thickness (cIMT) and carotid plaque 
1 (CP) were the proposed biomarkers of subclinical atherosclerosis associated with stroke

2 risk [2, 3]. Moreover, the vulnerable plaque of CAS can lead to the occurrence of

3 cerebral infarction [4]. Interestingly, as an important component of vascular structure,

4 excessive proliferation and inappropriate migration of vascular smooth muscle cells are

5 key factors in the formation of atherosclerotic plaque [5]. Recent studies have shown

6 that oxidized low-density lipoprotein (ox-LDL) can be used to simulate atherosclerosis

7 through exciting vascular smooth muscle cells [6]. Hence, repairing the dysfunction of

8 vascular smooth muscle cells might be an effective way to mitigate atherosclerosis

9 progression.

10 In recent years, long non-coding RNAs (lncRNAs), a class of transcripts with about 11200 nucleotides (nts), have been reported to play essential regulatory roles in multiple 12 biological and pathological activities by regulating diverse molecules, such as DNA, 13 RNA and proteins [7]. In fact, accumulating evidence suggested that abnormal 14 expression of IncRNAs is involved in the development and progression of numerous 15 diseases, including atherosclerosis [8, 9]. As Cai et al. reported, that abundance of 16 IncRNA TNK2-AS1 contributed to cell proliferation and migration of human aortic 17 smooth muscle cells (hASMCs) through inhibiting miR-150-5p and enhancing VEGFA 18 and FGF1 expression in atherosclerosis [10]. Analogously, Ji et al. confirmed that excess of linc-ROR aggravated the malignancy of atherosclerosis by increasing hASMCs proliferation and migration through regulating miR-195-5p/FGF2 axis [11].

21 LncRNA small nucleolar RNA host gene 16 (lnc-SNHG16) has been confirmed to work as an oncogene in a variety of cancers, such as bladder cancer [12], gastric cancer [13] and hepatocellular carcinoma [14]. Moreover, a recent literature manifested that lncSNHG16 could accelerate proliferation and inflammatory response of macrophages by interacting with miR17-5p to activate $\mathrm{NF}-\kappa \mathrm{B}$ signaling in atherosclerosis [15]. However, the underlying mechanism of lnc-SNHG16 in CAS is still unclear.

During the past decades, microRNAs (miRNAs), endogenous non-coding RNAs with 19-25 nts, have been shown to negatively regulate gene expression at posttranscriptional levels [16]. An extensive body of recent studies has proved that miRNAs could exert the regulating role in the progression of atherosclerosis [17, 18]. MicroRNA-30c-5p (miR-30c-5p) has been identified as a tumor suppressor by modulating target genes in gastric cancer [19], clear cell renal cell carcinoma [20] and multiple myeloma [21]. Furthermore, relevant studies have indicated that the low 
1 expression of miR-30c-5p, as an underlying predictive biomarker, was related to the

2 development of carotid intima-media thickness and early plaque in atherosclerosis [22,

3 23], implying that miR-30c-5p played a crucial function in the development of

4 atherosclerosis.

5 A disintegrin and metalloproteinase 10 (ADAM10), a member of the metalloprotease 6 family, takes part in the incision of diverse cells surface molecules, containing 7 cytokines, growth factors and adhesion molecules [24]. Simultaneously, some research

8 have confirmed that the knockdown of ADAM10 could weaken atherosclerosis 9 progression by suppressing inflammatory response and boosting cholesterol efflux [25]. 10 Yet, the specific role of ADAM10 in CAS remains unknown.

11 Herein, our results presented that lnc-SNHG16 was upregulated in CAS patient and 12 ox-LDL-treated hASMCs. Lnc-SNHG16 silencing hindered cell viability, migration, 13 inflammation, facilitated differentiation in ox-LDL-treated hASMCs. Moreover, 14 bioinformatics analysis suggested that miR-30c-5p had some sequences with lncSNHG16. Therefore, we aimed to investigate whether lnc-SNHG16 might regulate oxLDL- induced hASMCs damage through the miR-30c-5p/ADAM10 axis in CAS.

\section{Materials and Methods}

This research obtained the approval of Ethics Committee of The Third People's Hospital of Heze, and written informed consent was signed by each participant from The Third People's Hospital of Heze. Serum samples of the CAS patient $(n=30)$ were collected from patients with ischaemic stroke in the internal carotid artery underwent magnetic resonance angiography within 1 week of symptom onset while the central plaque and the interior layer of the outer plaque are relatively stable, and healthy volunteers $(n=30)$ were acquired from healthy volunteers. All the patients had no

27 cardioembolic stroke, haemorrhagic stroke, radiation therapy of the neck.

Human aortic smooth muscle cells (hASMCs) were provided by ScienCell (Carlsbad, $29 \mathrm{CA}$, USA), and were maintained in $5 \% \mathrm{CO}_{2}$ at $37^{\circ} \mathrm{C}$ under moist atmosphere with Dulbecco's modified Eagle's medium (DMEM; Hyclone, Beijing, China). 
1 Noteworthily, 10\% fetal bovine serum (FBS; Hyclone) and 1\% penicillin/streptomycin

2 as the supplement were added the culture medium. Besides, hASMCs were treated with

$30 \mathrm{ng} / \mathrm{mL}, 25 \mathrm{ng} / \mathrm{mL}, 50 \mathrm{ng} / \mathrm{mL}, 75 \mathrm{ng} / \mathrm{mL}$ and $100 \mathrm{ng} / \mathrm{mL}$ ox-LDL (Sigma-Aldrich,

4 St.Louis, MO, USA) for $24 \mathrm{~h}$ before measurement.

5

6 Real-time quantitative polymerase chain reaction (RT-qPCR)

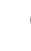

miR-30c-5p:

$$
\text { 5'-GCCGCTGTAAACATCCTACACT-3' }
$$

(sense), 5'-

GTGCAGGGTCCGAGGT-3’ (antisense); ADAM10: $\quad$ 5'-AAGAAGCTTCCCACAAGGCA-3' (sense), 5'TGTGTACGCAGAGTATCTAACTGG-3' (antisense);

U6: 5'-CTCGCTTCGGCAGCACA-3' (sense), 5'-AACGCTTCACGAATTTGCGT-3' (antisense);

GAPDH: $\quad$ 5'-GTCAACGGATTTGGTCTGTATT-3' $\quad$ (sense), 5'AGTCTTCTGGGTGGCAGTGAT-3' (antisense). 
1 Lnc-SNHG16 small interference RNA (si-lnc-SNHG16) and its negative control (si-

$2 \mathrm{NC}$ ), miR-30c-5p inhibitor (anti-miR-30c-5p) and its negative control (anti-miR-NC),

3 miR-135a-5p mimics (miR-135a-5p) and its negative control (miR-NC) were acquired

4 from RiboBio (Guangzhou, China). The pcDNA3.1 vector (pcDNA, Addgene,

5 Cambridge, MA, USA) was applied to construct lnc-SNHG16 and ADAM10

6 overexpression vector, termed as pcDNA-lnc-SNHG16 (lnc-SNHG16) and pcDNA-

7 ADAM10 (ADAM10). With the help of Lipofectamine 3000 reagent (Invitrogen), these

8 oligonucleotides and plasmids were transfected into hASMCs. After $24 \mathrm{~h}$ of

9 transfection, transfected cells were harvested and used for the following experiments.

11 Cell viability assay

12 Cell viability of hASMCs was detected by using 3-(4, 5-dimethyl-2-thiazolyl)-2, 513 diphenyl-2-H-tetrazolium bromide (MTT, Sigma-Aldrich) referring to the supplier's 14 direction. In brief, hASMCs were seeded on 96 -well plates $\left(5 \times 10^{3}\right.$ cells/well $)$. After 15 treatment with ox-LDL for $24 \mathrm{~h}, 20 \mu \mathrm{L}$ MTT (Sigma-Aldrich) was added into each well 16 at indicated time points $(0 \mathrm{~h}, 24 \mathrm{~h}, 48 \mathrm{~h}$ and $72 \mathrm{~h})$, followed by incubation for another $174 \mathrm{~h}$ at $37^{\circ} \mathrm{C}$. Whereafter, the supernatant was discarded, $150 \mu \mathrm{L}$ of dimethyl sulfoxide 18 (DMSO, Sigma-Aldrich) was added to terminate the reaction. At last, with the help of 19 microplate reader (Thermo Electron Corporation, Vantaa, Finland), cell absorbance 20 was read at $490 \mathrm{~nm}$.

Migration ability of hASMCs was evaluated in accordance with the instructions of 24 Transwell chambers (Corning Incorporated, Corning, NY, USA). Generally, the treated hASMCs $\left(1 \times 10^{5}\right)$ in serum-free medium were introduced into the upper chamber of transwell, and the medium with 10\% FBS (Hyclone) was added in the lower chamber. After incubation for $24 \mathrm{~h}$, cells remaining on the upper surface of membranes were scraped with cotton swabs, while cells migrated to the lower surface of membranes were fixed in methanol and stained by crystal violet. Finally, an inverted microscope was used to count the number of stained cells. 
5

In this assay, hASMCs were incubated in 24-well plates. After treatment with oxLDL for $24 \mathrm{~h}$, the medium was collected, followed by detection the levels of interleukin-6 (IL-6), IL- $\beta$ and tumor necrosis factor- $\alpha$ (TNF- $\alpha$ ) with an ELISA (Beyotime, Shanghai, China) kits based on the user's guidebook. A SpectraMaxM2 microplate reader (Molecular Devices, Sunnyvale, CA, USA) was applied for the measurement of the absorbance in plates.

\section{Western blot assay}

In brief, total protein from serums and cells was extracted by using RIPA buffer with protease and phosphates inhibitions (Sigma-Aldrich), and the concentrations were quantified with BCA Protein Assay Kit (Beyotime, China). After separation with a sodium dodecyl sulfate-polyacrylamide gel electrophoresis (SDS-PAGE), the protein samples were transferred onto polyvinylidene fluoride (PVDF) membranes (Millipore, Bedford, MA, USA), followed by blocking with 5\% skim milk. After incubation for 2 $\mathrm{h}$, the primary antibodies were incubated with the membranes at $4^{\circ} \mathrm{C}$. The next day, the corresponding horseradish peroxidase (HRP)-conjugated secondary antibodies were further cultured in the membranes. Finally, an enhanced chemiluminescence kit (ECL; Amersham Biosciences, Pittsburg, PA, Sweden) was conducted to analyze these protein signals. Primary antibodies were as followed: anti-spinal muscular atrophy (anti-SMA; 1:1000, ab5694, Abcam, Cambridge, MA, USA), anti-calponin (1:1000, ab46794, Abcam), anti-ADAM10 (1:1000, ab124695, Abcam) and anti-GAPDH (1:1000, ab8227, Abcam).

7 Lnc-SNHG16 wild-type reporter vector (lnc-SNHG16 WT) containing the binding sites for miR-30c-5p and its mutant-type in complementary sites (lnc-SNHG16 MUT), 9 ADAM10 3' un-translated region (3'UTR) wild-type reporter vector (WT ADAM10 3'UTR) possessing the binding sequences with miR-30c-5p and its mutant-type in seed 
1 region (MUT ADAM10 3'UTR) were amplified and then sub-cloned into pMIR-

$2 \mathrm{GLO}^{\mathrm{TM}}$ vector (Promega, Madison, WI, USA). Subsequently, according to the standard

3 method of Lipofectamine 3000 (Invitrogen), hASMCs in 48-well plates were co-

4 transfected with the constructed reporter plasmids and miR-135a-5p or miR-NC.

5 Luciferase activities at $48 \mathrm{~h}$ after transfection were detected under a dual-luciferase

6 reporter assay kit (Promega).

7

8 Statistical analysis

9 Statistical analysis was conducted with GraphPad Prism7 software, and was shown 10 as the mean \pm standard deviation (SD). Differences between two groups were analyzed by using Student's t-test. Differences between more than two groups were identified using one-way analysis of variance (ANOVA) followed by Tukey's tests. All assays were carried out at least in triplicate. If $P$ value $<0.05$, it was regarded as statistically significant.

\section{Results}

Lnc-SNHG16 expression was increased and miR-30c-5p expression was decreased in CAS patient and ox-LDL-treated HASMCs

At first, to investigate the function of lnc-SNHG16 and miR-30c-5p in CAS, their expression levels were detected through RT-qPCR assay. Compared with the serums of healthy volunteers $(n=30)$, lnc-SNHG16 was highly expressed and miR-30c-5p was lowly expressed in CAS patient serums ( $\mathrm{n}=30$ ) (Figure 1A and 1C). Importantly, we found that lnc-SNHG16 expression was improved, and miR-30c-5p expression was reduced in hASMCs with the increase of ox-LDL concentration, when compared to cells un-treated with ox-LDL (Figure 1B and 1D), suggesting that the involvement of lnc-SNHG16 and miR-30c-5p in the ox-LDL induced CAS model. Particularly in cells treated with $50 \mathrm{ng} / \mathrm{mL}$ ox-LDL, hence, we chose $50 \mathrm{ng} / \mathrm{mL}$ ox-LDL $24 \mathrm{~h}$ for subsequent experiments. Furthermore, lnc-SNHG16 level was inversely related to miR-30c-5p expression in CAS patient serums (Figure 1E). In a word, the dysregulation of lncSNHG16 and miR-30c-5p might be correlated with CAS. 
2 Lnc-SNHG16 knockdown repressed cell viability, migration, inflammation,

3 facilitated differentiation in ox-LDL-treated hASMCs

4 Then, to explore the role of lnc-SNHG16 in CAS, we knocked down lnc-SNHG16 5 expression in ox-LDL treated hASMCs. As displayed in Figure 2A, in the treatment of 6 hASMCs, lnc-SNHG16 level was markedly declined in si-lnc-SNHG16-transfected 7 relative to cells transfected with si-NC. Functional analysis showed that the promotion 8 of cell viability and migration caused by ox-LDL was obviously attenuated through lnc9 SNHG16 downregulation (Figure 2B and 2C). Synchronously, introduction of si-lnc10 SNHG16 evidently diminished the enhancement of ox-LDL on inflammatory factors 11 (IL-6, IL- $\beta$ and TNF- $\alpha$ ) level (Figure 2D), indicating that lnc-SNHG16 silencing 12 retarded the inflammation of ox-LDL induced CAS model. Apart from that, lnc13 SNHG16 deletion abrogated ox-LDL-triggered decrease in the protein levels of 14 differentiation-related factors (SMA and Calponin) in hASMCs (Figure 2E), proving 15 that lnc-SNHG16 deficiency accelerated the differentiation of ox-LDL induced CAS model. Collectively, these results suggested that the knockdown of lnc-SNHG16 impeded cell viability, migration, inflammation, and expedited differentiation in oxLDL-treated hASMCs.

MiR-30c-5p was direct target of lnc-SNHG16

Given that there was a negative correlation between lnc-SNHG16 and miR-30c-5p in CAS patient serum. We used to the bioinformatics software starBase to further analyze the underlying relationship between lnc-SNHG16 and miR-30c-5p. As presented in Figure 3A, miR-30c-5p was found to harbor some common complementary sequences with lnc-SNHG16. To verify the predicted results, a dualluciferase reporter assay was conducted in hASMCs. Data showed that the overexpression of miR-30c-5p reduced the luciferase activity of WT-lnc-SNHG16 reporter vector, but not that of MUT-lnc-SNHG16 reporter vector (Figure 3B). Moreover, the transfection efficiency of pcDNA-lnc-SNHG16 was detected and exhibited in Figure 3C. And lnc-SNHG16 knockdown contributed to miR-30c-5p level, and lnc-SNHG16 overexpression suppressed miR-30c-5p level in ox-LDL-treated 
1 hASMCs (Figure 3D). Notably, re-introduction of anti-miR-30c-5p abolished the

2 facilitation of lnc-SNHG16 knockdown on miR-30c-5p level in ox-LDL-treated 3 hASMCs (Figure 3E). Functionally, deletion of miR-30c-5p notably abated the 4 inhibitory effect of lnc-SNHG16 downregulation on cell viability and migration in ox5 LDL-induced hASMCs (Figure 3F and 3G). Besides, the reduction of IL-6, IL- $\beta$ and 6 TNF- $\alpha$ levels due to lnc-SNHG16 deficiency was undermined by miR-30c-5p 7 knockdown in ox-LDL-stimulated hASMCs (Figure 3H), implying that silencing of 8 miR-30c-5p overturned the suppression effect of lnc-SNHG16 downregulation on inflammatory response in ox-LDL-excited hASMCs. Western blot assay confirmed that anti-miR-30c-5p reversed the positive effect of lnc-SNHG16 knockdown on differentiation in ox-LDL-provoked hASMCs, showing that the protein levels of SMA and Calponin increased by si-lnc-SNHG16 was repressed through miR-30c-5p downregulation in ox-LDL-treated hASMCs (Figure 3I). All these results unveiled that miR-30c-5p, as a target of lnc-SNHG16, partially abolished the effects of lnc-SNHG16 on cell viability, migration, inflammation, and differentiation in ox-LDL-trigger hASMCs.

As widely believed, miRNAs could exert the function by binding to the 3'untranslated regions (3'UTR) of mRNAs [28]. Thus, to further explore the mechanism of miR-30c-5p, we searched the latent target genes of miR-30c-5p by using the bioinformatics tool (StarBase). Results presented that miR-30c-5p contained a motif with sites complementary to ADAM10 3'UTR (Figure 4A). Then, we preformed the dual-luciferase reporter assay to further confirm the direct interaction between miR30c-5p and ADAM10 3'UTR. As showed in Figure 4B, miR-30c-5p upregulation led to an overt decline in luciferase activity of WT-ADAM10 3'UTR, while had little effect on MUT-ADAM10 3'UTR in hASMCs. In addition, ADAM10 was expressed at the high level in both CAS patient serum (Figure 4C and 4D) and ox-LDL-treated hASMCs (Figure 4E and 4F) in comparison with their respective control groups. And transfection efficiency of anti-miR-30c-5p and miR-30c-5p mimics were examined and shown in

31 Figure 4H. Intriguingly, we viewed that there was an inverse relationship between miR- 
1 improved the expression level of ADAM10, whereas miR-30c-5p upregulation declined ADAM10 level in ox-LDL-stimulated hASMCs (Figure 4I and 4J). Taken together, these findings suggested that miR-30c-5p directly bound with ADAM10.

4

MiR-30c-5p-mediated cell viability, migration, inflammation and differentiation were reversed by ADAM10 in ox-LDL-treated hASMCs

As mentioned above, ADAM10 was a direct target of miR-30c-5p in ox-LDL-treated hASMCs. Meanwhile, ADAM10 as a carcinogenic factor has been testified by previous studies $[29,30]$. We inferred that miR-30c-5p could exert the suppressive effect on oxLDL induced CAS model by interacting with ADAM10. Firstly, the transfection efficiency of pcDNA-ADAM10 was detected by RT-qPCR and western blot assays in ox-LDL-induced hASMCs. As illustrated in Figure 5A and 5B, ADAM10 level was augmented in ADAM10-transfected hASMCs versus cells transfected with pcDNA. Importantly, rescue assays rendered that ADAM10 overexpression overturned the inhibiting effect of miR-30c-5p upregulation on cell viability and migration in ox-LDLstimulated hASMCs (Figure 5C and 5D). Consistently, re-transfection of pcDNAADAM10 evidently abrogated miR-30c-5p mimics-caused decrease in the levels of IL6 , IL- $\beta$ and TNF- $\alpha$ in ox-LDL-treated hASMCs, supporting that ADAM10 upregulation partly reversed the adverse effect of miR-30c-5p overexpression on inflammatory response in ox-LDL-induced hASMCs (Figure 5E). Additionally, reduced SMA and Calponin protein levels further confirmed that upregulation of ADAM10 could effectively abolished the positive effect of miR-30c-5p mimics on differentiation in oxLDL-triggered hASMCs (Figure 5F). Overall, these results suggested that miR-30c-5p could regulate cell viability, migration, inflammation, and differentiation of ox-LDLtrigger hASMCs by targeting ADAM10.

Verification of lnc-SNHG16/miR-30c-5p/ADAM10 regulatory axis in ox-LDLtreated hASMCs

Based on the above results, we conjectured that lnc-SNHG16 could exert its regulatory role by the miR-30c-5p/ADAM10 axis in ox-LDL-treated hASMCs. To testify the guess, we implemented the rescue assays to further verify whether lnc- 
1 SNHG16 affect ADAM10 expression through miR-30c-5p. As shown in Figure 6, antimiR-30c-5p improved the protein level of ADAM10, while lnc-SNHG16 knockdown could relieve miR-30c-5p silencing-mediated promotion effect on ADAM10 protein level in ox-LDL-induced hASMCs. Together, the results unveiled that lnc-SNHG16 could perform as a molecular sponge of miR-30c-5p to affect ADAM10 expression.

\section{Discussion}

Increasing evidence exhibits that lncRNAs can be used as prognostic biomarkers in multiple diseases, including atherosclerosis [31]. Indeed, as the research moves along, lncRNAs have become necessary regulators in the development and progression of atherosclerosis [32-35]. Lnc-SNHG16, located at 17q25.1 gene, was identified as an oncogenic lncRNA in various types of cancer [36, 37]. Importantly, in a recent publication, lnc-SNHG16 was verified to be abnormally increased and exacerbated the progression of atherosclerosis through triggering hASMCs proliferation and migration [38]. However, the underlying mechanism of lnc-SNHG16 in CAS still needs further clarification. In this study, the different concentration of ox-LDL induced the aortic smooth muscle cells (hASMCs) in an Atherosclerosis simulation environment was used for further research. Lnc-SNHG16 was upregulated in CAS patient serum samples and ox-LDL-treated hASMCs cells, suggesting that lnc-SNHG16 might involve in CAS progression. Functionally, lnc-SNHG16 deficiency constrained ox-LDL-induced proliferation, migration, the expression of inflammatory factor and differentiation of hASMCs, demonstrating that lnc-SNHG16 mitigated the progression of CAS.

MiR-30c-5p, a tumor suppressor, has been pointed out to exert the suppressive action in atherosclerosis development [39]. In this manuscript, our data showed that miR-30c$5 \mathrm{p}$ was decreased in CAS patient and ox-LDL-treated hASMCs. Intriguingly, there was an inverse relation between miR-30c-5p and lnc-SNHG16 in CAS. Previous studies have suggested that lncRNAs could exert the function through the interaction with miRNAs [40]. Our results first confirmed that miR-30c-5p was a direct target of lncSNHG16, and miR-30c-5p downregulation could relieve the negatively effect of lncSNHG16 deletion on ox-LDL-excited hASMCs damage. The inhibitory action of miR$30 c-5 p$ on ox-LDL-caused cell injury of atherosclerosis was also testified in prior report 
1 It is widely accepted that lncRNAs could impact mRNA expression by sponging miRNA [42]. ADAM10 as the target of miR-30c-5p was first validated in hASMCs. Apart from that, some reports have presented that ADAM10 was closely linked to the composition of atherosclerosis plaque [43, 44]. In this research, ADAM10 was upregulated in CAS patient and ox-LDL-treated hASMCs, and partly reversed the suppression effect of miR-30c-5p on ox-LDL-caused hASMCs damage. Consistent with our data, ADAM10 could facilitate the inflammatory response in atherosclerosis [25]. Additionally, mechanistic analysis further verified that the downregulation of lncSNHG16 could overturn the positive action of miR-30c-5p knockdown on ADAM10 expression in ox-LDL-treated hASMCs. That was to say, lnc-SNHG16 preformed as a sponge of miR-30c-5p to upregulate ADAM10 expression, thereby regulating CAS progression.

While the present results highlights the regulation roles of lnc-SNHG16 in CAS, we should note the limitation of this research. One limitation of the present research is, although our initial comparison of the miRNA and circRNA expression level in normal and CAS serum samples was credible, the limited sample size makes further evaluation necessary. Another himation is our research was limited to an in vitro study, therefore, an in vivo experiments will be seriously considered in the future. Besides, whether SNHG16 correlates with the progression of atherosclerosis and the effects of targeting SNHG16 on therapy for atherosclerosis may require further investigation.

21 Together, this study first discovered the regulatory role of the lnc-SNHG16/miR30c-5p/ADAM10 axis in the ox-LDL induced CAS model. Our findings provided an insight into the molecular basis of carotid atherosclerosis, which will provide us important clues for developing the effective therapeutic strategies.

Acknowledgement

None 
1 The authors declare that they have no financial conflicts of interest

2

\section{Funding}

$4 \quad$ None

5

6 


\section{REFERENCES}

2 1. de Weerd M, Greving JP, Hedblad B, et al. Prevalence of asymptomatic carotid

3 artery stenosis in the general population: an individual participant data meta-analysis.

$4 \quad$ Stroke 2010; 41: 1294-7.

5 2. Rundek T, Gardener H, Della-Morte D, et al. The relationship between carotid

6 intima-media thickness and carotid plaque in the Northern Manhattan Study.

7 Atherosclerosis 2015; 241: 364-70.

8 3. Ammirati E, Moroni F, Norata GD, et al. Markers of inflammation associated with

9 plaque progression and instability in patients with carotid atherosclerosis. Mediators

10 Inflamm 2015; 2015: 718329.

11 4. Lin K, Zhang ZQ, Detrano R, et al. Carotid vulnerable lesions are related to 12 accelerated recurrence for cerebral infarction magnetic resonance imaging study. Acad 13 Radiol 2006; 13: 1180-6.

14 5. Low EL, Baker AH, Bradshaw AC. TGFbeta, smooth muscle cells and coronary 15 artery disease: a review. Cell Signal 2019; 53: 90-101.

16 6. Cao L, Zhang Z, Li Y, et al. LncRNA H19/miR-let-7 axis participates in the 17 regulation of ox-LDL-induced endothelial cell injury via targeting periostin. Int Immunopharmacol 2019; 72: 496-503. 7. Wapinski O, Chang HY. Long noncoding RNAs and human disease. Trends Cell 20 Biol 2011; 21: 354-61.

21 8. Jian L, Jian D, Chen Q, Zhang L. Long Noncoding RNAs in Atherosclerosis. J 22 Atheroscler Thromb 2016; 23: 376-84.

23 9. Arslan S, Berkan O, Lalem T, et al. Long non-coding RNAs in the atherosclerotic 24 plaque. Atherosclerosis 2017; 266: 176-81.

25 10. Cai T, Cui X, Zhang K, et al. LncRNA TNK2-AS1 regulated ox-LDL-stimulated 26 HASMC proliferation and migration via modulating VEGFA and FGF1 expression by sponging miR-150-5p. 2019; 23: 7289-98.

28 11. Ji Z, Chi J, Sun H, et al. Linc-ROR targets FGF2 to regulate HASMC proliferation 29 and migration via sponging miR-195-5p. Gene 2020; 725: 144143.

30 12. Cao X, Xu J, Yue D. LncRNA-SNHG16 predicts poor prognosis and promotes 31 tumor proliferation through epigenetically silencing p21 in bladder cancer. Cancer 32 Gene Ther 2018; 25: 10-7. 
1 13. Lian D, Amin B, Du D, Yan W. Enhanced expression of the long non-coding RNA

2 SNHG16 contributes to gastric cancer progression and metastasis. Cancer Biomark

$3 \quad 2017 ; 21: 151-60$.

4 14. Chen H, Li M, Huang P. LncRNA SNHG16 Promotes Hepatocellular Carcinoma

5 Proliferation, Migration and Invasion by Regulating miR-186 Expression. J Cancer $62019 ; 10: 3571-81$.

7 15. An JH, Chen ZY, Ma QL, et al. LncRNA SNHG16 promoted proliferation and

8 inflammatory response of macrophages through miR-17-5p/NF-kappaB signaling

9 pathway in patients with atherosclerosis. Eur Rev Med Pharmacol Sci 2019; 23: 86651077.

11 16. Bartel DP. MicroRNAs: target recognition and regulatory functions. Cell 2009;

12 136: 215-33.

13 17. Lu Y, Thavarajah T, Gu W, et al. Impact of miRNA in Atherosclerosis. Arterioscler 14 Thromb Vasc Biol 2018; 38: e159-e70.

15 18. Brennan E, Wang B, McClelland A, et al. Protective Effect of let-7 miRNA Family 16 in Regulating Inflammation in Diabetes-Associated Atherosclerosis. Diabetes 2017; 17 66: 2266-77.

18 19. Cao JM, Li GZ, Han M, et al. MiR-30c-5p suppresses migration, invasion and 19 epithelial to mesenchymal transition of gastric cancer via targeting MTA1. Biomed 20 Pharmacother 2017; 93: 554-60.

21 20. Song S, Long M, Yu G, et al. Urinary exosome miR-30c-5p as a biomarker of clear 22 cell renal cell carcinoma that inhibits progression by targeting HSPA5. J Cell Mol Med 23 2019; 23: 6755-65.

24 21. Zhao JJ, Lin J, Zhu D, et al. miR-30-5p functions as a tumor suppressor and novel 25 therapeutic tool by targeting the oncogenic Wnt/beta-catenin/BCL9 pathway. Cancer 26 Res 2014; 74: 1801-13.

27 22. Relevance of low miR-30c-5p levels in atherogenesis: a promising predictive 28 biomarker and potential therapeutic target. Cardiovasc Res 2018; 114: 258.

29 23. Ceolotto G, Giannella A, Albiero M, et al. miR-30c-5p regulates macrophage30 mediated inflammation and pro-atherosclerosis pathways. Cardiovasc Res 2017; 113: 31 1627-38.

32 24. Saint-Pol J, Eschenbrenner E, Dornier E, et al. Regulation of the trafficking and 33 the function of the metalloprotease ADAM10 by tetraspanins. Biochem Soc Trans 34 2017; 45: 937-44. 
1 25. Li H, Han S, Sun Q, et al. Long non-coding RNA CDKN2B-AS1 reduces inflammatory response and promotes cholesterol efflux in atherosclerosis by inhibiting ADAM10 expression. Aging (Albany NY) 2019; 11: 1695-715.

26. Puch-Hau C, Sanchez-Tapia IA, Patino-Suarez V, et al. Evaluation of two independent protocols for the extraction of DNA and RNA from different tissues of sea cucumber Isostichopus badionotus. MethodsX 2019; 6: 1627-34. 27. Livak KJ, Schmittgen TD. Analysis of relative gene expression data using realtime quantitative PCR and the 2(-Delta Delta C(T)) Method. Methods 2001; 25: 402-8. 28. Huntzinger E, Izaurralde E. Gene silencing by microRNAs: contributions of translational repression and mRNA decay. Nat Rev Genet 2011; 12: 99-110. 29. Guo H, Yang S, Li S, et al. LncRNA SNHG20 promotes cell proliferation and invasion via miR-140-5p-ADAM10 axis in cervical cancer. Biomed Pharmacother 2018; 102: 749-57.

30. Sun SQ, Ren LJ, Liu J, et al. Sevoflurane inhibits migration and invasion of colorectal cancer cells by regulating microRNA-34a/ADAM10 axis. Neoplasma 2019; 2019.

31. Li H, Zhu H, Ge J. Long Noncoding RNA: Recent Updates in Atherosclerosis. Int J Biol Sci 2016; 12: 898-910.

32. Zhang Z, Salisbury D, Sallam T. Long Noncoding RNAs in Atherosclerosis: JACC Review Topic of the Week. J Am Coll Cardiol 2018; 72: 2380-90.

33. Archer K, Broskova Z, Bayoumi AS, et al. Long Non-Coding RNAs as Master Regulators in Cardiovascular Diseases. Int J Mol Sci 2015; 16: 23651-67.

34. Pan JX. LncRNA H19 promotes atherosclerosis by regulating MAPK and NF-kB signaling pathway. Eur Rev Med Pharmacol Sci 2017; 21: 322-8.

35. Yan L, Liu Z, Yin H, et al. Silencing of MEG3 inhibited ox-LDL-induced inflammation and apoptosis in macrophages via modulation of the MEG3/miR204/CDKN2A regulatory axis. Cell Biol Int 2019; 43: 409-20.

36. Zhu H, Zeng Y, Zhou CC, Ye W. SNHG16/miR-216-5p/ZEB1 signal pathway contributes to the tumorigenesis of cervical cancer cells. Arch Biochem Biophys 2018; 637: 1-8. Sponging MiR-4518 and Up-Regulating PRMT5 Expression in Glioma. Cell Physiol 
1 38. Lin Y, Tian G, Zhang H, et al. Long non-coding RNA SNHG16 regulates human aortic smooth muscle cell proliferation and migration via sponging miR-205 and modulating Smad2. 2019; 23: 6919-29. 39. Li P, Zhong X, Li J, et al. MicroRNA-30c-5p inhibits NLRP3 inflammasomemediated endothelial cell pyroptosis through FOXO3 down-regulation in atherosclerosis. Biochem Biophys Res Commun 2018; 503: 2833-40. 40. Jalali S, Bhartiya D, Lalwani MK, et al. Systematic transcriptome wide analysis of lncRNA-miRNA interactions. PLoS One 2013; 8: e53823. inflammatory response and apoptosis of HUVEC by lncRNA XIAT knockdown via regulating miR-30c-5p/PTEN axis. Eur Rev Med Pharmacol Sci 2019; 23: 7628-38. 42. He L, Chen Y, Hao S, Qian J. Uncovering novel landscape of cardiovascular diseases and therapeutic targets for cardioprotection via long noncoding RNA-miRNAmRNA axes. Epigenomics 2018; 10: 661-71. 43. van der Vorst EP, Jeurissen M, Wolfs IM, et al. Myeloid A disintegrin and metalloproteinase domain 10 deficiency modulates atherosclerotic plaque composition by shifting the balance from inflammation toward fibrosis. Am J Pathol 2015; 185 : 1145-55. 44. Donners MM, Wolfs IM, Olieslagers S, et al. A disintegrin and metalloprotease 10 is a novel mediator of vascular endothelial growth factor-induced endothelial cell function in angiogenesis and is associated with atherosclerosis. Arterioscler Thromb Vasc Biol 2010; 30: 2188-95.

Figure Legends

Figure 1 Lnc-SNHG16 was upregulated and miR-30c-5p was downregulated in CAS and ox-LDL-treated hASMCs. (A and C) RT-qPCR assay was preformed to detect the expression level of lnc-SNHG16 and miR-30c-5p in 30 pairs of CAS patient 
1 miR-30c-5p in hASMCs treated with ox-LDL at different concentrations $(0 \mathrm{ng} / \mathrm{mL}, 25$ $\mathrm{ng} / \mathrm{mL}, 50 \mathrm{ng} / \mathrm{mL}, 75 \mathrm{ng} / \mathrm{mL}$ and $100 \mathrm{ng} / \mathrm{mL}$ ). (D) The expression association between lnc-SNHG16 and miR-30c-5p in CAS patients was analyzed by Pearson correlation analysis. ${ }^{*} P<0.05$.

Figure 2 Lnc-SNHG16 knockdown suppressed cell viability, migration, inflammation, promoted differentiation in ox-LDL-treated hASMCs. (A) LncSNHG16 level was measured by RT-qPCR assay in hASMCs treated with Control, oxLDL, ox-LDL + si-NC and ox-LDL + si-lnc-SNHG16. (B) Cell viability was detected by MTT assay in treated hASMCs. (C) Migration ability was assessed by transwell assay in treated hASMCs. (D) Expression levels of IL-6, IL- $\beta$ and TNF- $\alpha$ were examined by ELISA in treated hASMCs. (E) The protein levels of SMA and Calponin were tested by western blot assay in treated hASMCs. $* P<0.05$.

Figure 3 Lnc-SNHG16 directly bound with miR-30c-5p. (A) The binding sites between lnc-SNHG16 and miR-30c-5p were predicted by starBase software. (B) The effects of miR-30c-5p overexpression on luciferase activity of WT-lnc-SNHG16 and MUT-lnc-SNHG16 reporters in hASMCs were measured by dual-luciferase reporter assay. (C-I) HASMCs were stimulated with $50 \mathrm{ng} / \mathrm{mL}$ ox-LDL for $24 \mathrm{~h}$. (C) LncSNHG16 level was assessed in hASMCs transfected with Control, pcDNA and lncSNHG16. (D) MiR-30c-5p level was measured in hASMCs transfected with Control, si-NC, si-lnc-SNHG16, pcDNA and lnc-SNHG16. (E) MiR-30c-5p level was measured in hASMCs transfected with Control, si-NC, si-lnc-SNHG16, si-lnc-SNHG16 + antimiR-NC and si-lnc-SNHG16 + anti-miR-30c-5p. (F) Cell viability in treated hASMCs was tested by MTT assay. (G) Migration capacity in treated hASMCs was measured by transwell assay. (H) The levels of IL-6, IL- $\beta$ and TNF- $\alpha$ in treated hASMCs were by ELISA. (I) SMA and Calponin protein levels in treated hASMCs were western blot assay. ${ }^{*} P<0.05$.

Figure 4 ADAM10 was the target of miR-30c-5p. (A) StarBase software was applied to predict the binding sequence between miR-30c-5p and ADAM10 3'UTR. (B) The dual-luciferase reporter assay was conducted to confirm the binding relationship between miR-30c-5p and ADAM10 3'UTR in hASMCs. (C and D) The mRNA level and protein level of ADAM10 were detected in 30 pairs of CAS patient serum and healthy volunteer serum (E and F) ADAM10 level was measured in hASMCs 
1 stimulated with ox-LDL at various concentrations $(0 \mathrm{ng} / \mathrm{mL}, 25 \mathrm{ng} / \mathrm{mL}, 50 \mathrm{ng} / \mathrm{mL}, 75$ $\mathrm{ng} / \mathrm{mL}$ and $100 \mathrm{ng} / \mathrm{mL}$ ). (G) Pearson correlation analysis was preformed to appraise the expression association between miR-30c-5p and ADAM10 in CAS patients. (H-J) hASMCs were treated with $50 \mathrm{ng} / \mathrm{mL}$ ox-LDL for $24 \mathrm{~h}$. (H) MiR-30c-5p level was examined in hASMCs transfected with Control, anti-miR-NC, anti-miR-30c-5p, miRNC and miR-30c-5p. (I and J) The mRNA level and protein level of ADAM10 were

7 detected in treated hASMCs. $* P<0.05$.

8 Figure 5 ADAM10 abrogated the effects of miR-30c-5p on cell viability, migration, 9 inflammation and differentiation in ox-LDL-treated hASMCs. HASMCs were 10 treated with $50 \mathrm{ng} / \mathrm{mL}$ ox-LDL for $24 \mathrm{~h}$. (A and B) ADAM10 level was tested in 11 hASMCs transfected with Control, pcDNA and ADAM10. (C) MTT assay was used to 12 detect the proliferative ability in hASMCs transfected with Control, miR-NC, miR-30c$135 \mathrm{p}, \mathrm{miR}-30 \mathrm{c}-5 \mathrm{p}+\mathrm{pcDNA}$ and miR-30c-5p + ADAM10. (D) Transwell assay was 14 carried out to measure the migration in treated hASMCs. (E) ELISA was preformed to 15 assess the levels of IL-6, IL- $\beta$ and TNF- $\alpha$ in treated hASMCs. (F) Western blot assay 16 was applied to examine the protein levels of SMA and Calponin in treated HASMCs. $17 * P<0.05$.

Figure 6 Lnc-SNHG16 regulated ADAM10 expression through sponging miR-30c5p. HASMCs were treated with $50 \mathrm{ng} / \mathrm{mL}$ ox-LDL for $24 \mathrm{~h}$. ADAM10 protein level was detected with Control, anti-miR-NC, anti-miR-30c-5p, anti-miR-30c-5p + si-NC and anti-miR-30c-5p + si-lnc-SNHG16. $* P<0.05$. 
A

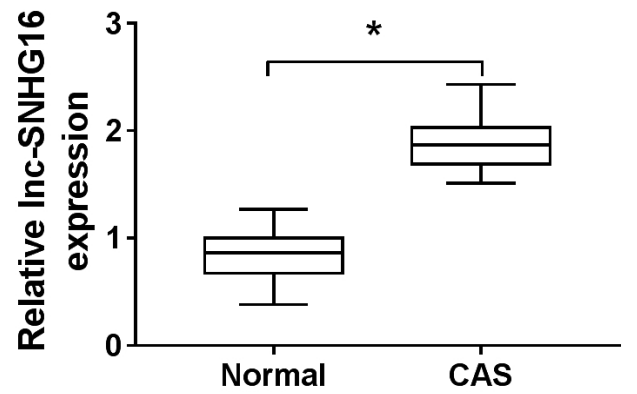

C

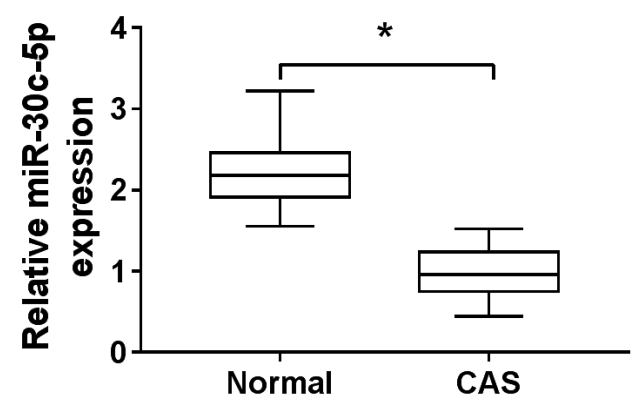

E

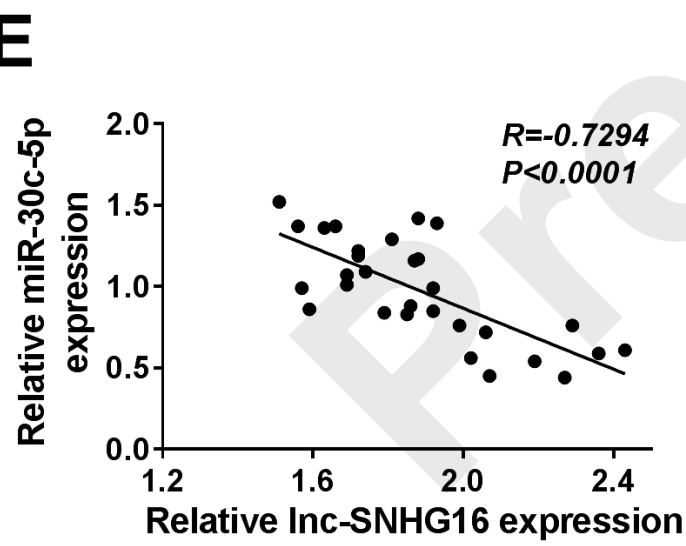

B

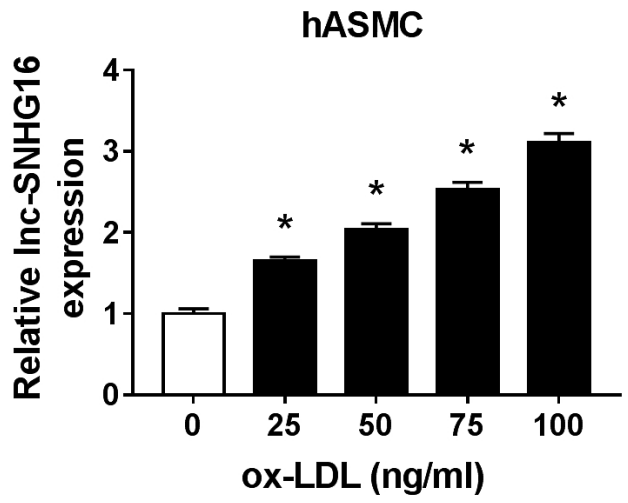

D

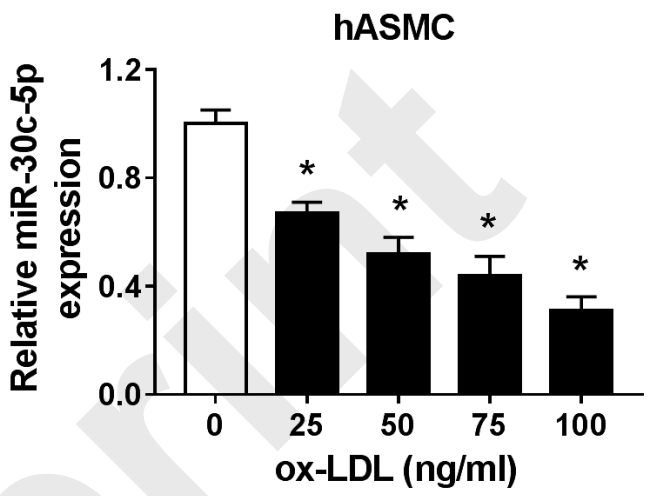



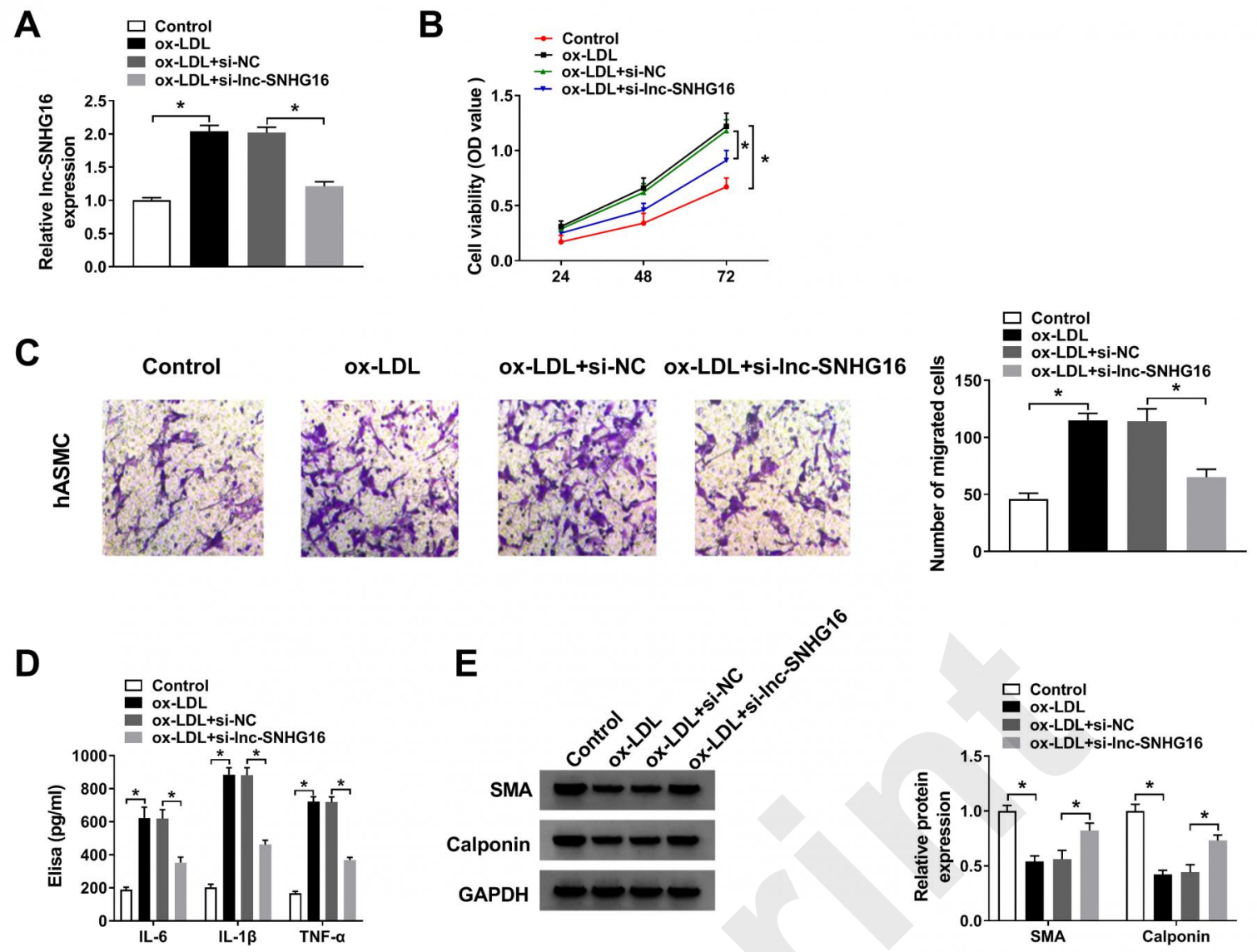
A

Inc-SNHG16 WT 5' UUCCUGUUUGUAAGAUUUGUUUACA 3' miR-30c-5p 3' CGACUCUCACAUCCU. - ACAAAUGU 5'

Inc-SNHG16 MUT 5' UUCCUGUUUGUAAGAUUACAAAUGA 3'

C

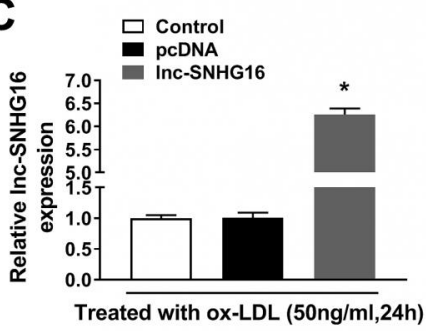

$\mathbf{F}$
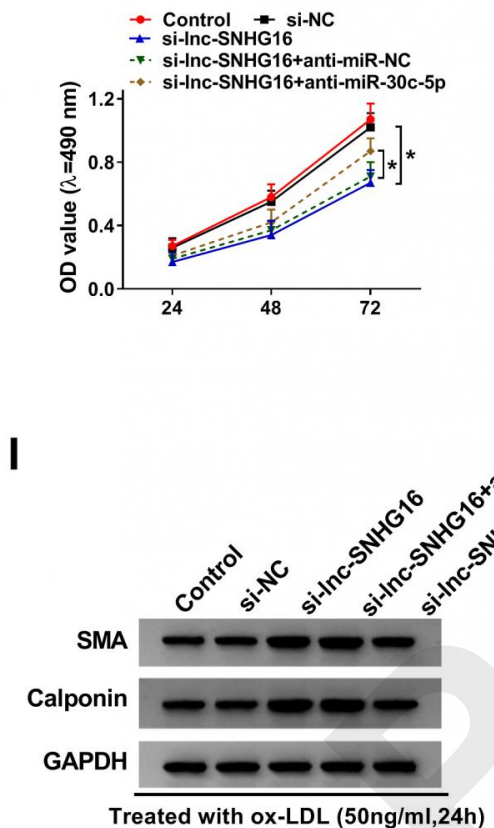

$\square$ Control

si-NC si-Inc-SNHG16

si-Inc-SNHG16+anti-miR-NC

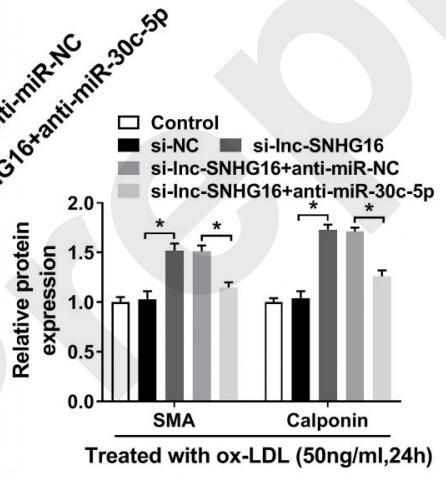

Treated with ox-LDL (50ng/ml,24h) | | | | | |

B

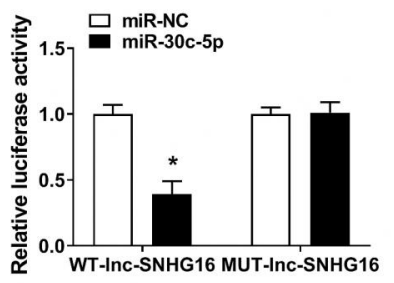

E $\square$ si-NC sontrol $_{\text {si-Inc-SNHG16 }}$

si-Inc-SNHG16+anti-miR-NC

D $\quad$ Control

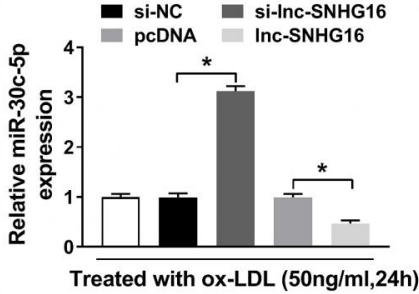

G

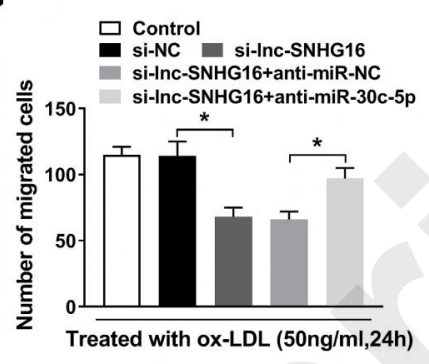

H

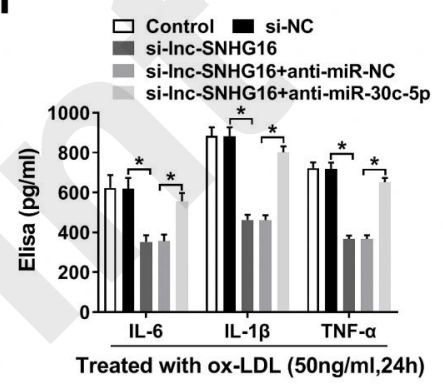


A

WT ADAM10 3'UTR 5' UUUAAGAGUAUA- - AGGUUUACA 3' miR-30c-5p 3' CGACUCUCACAUCCUACAAAUGU 5' MUT ADAM10 3'UTR 5' UUUAAGAGUAUA- - AGCAAAUGA 3'

C

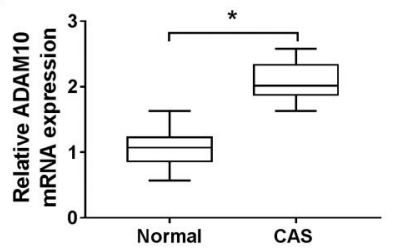

E

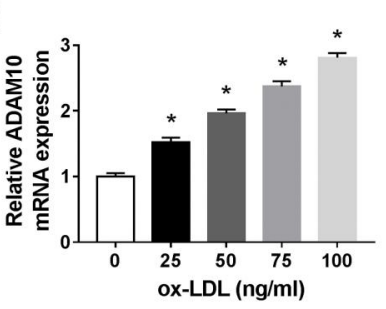

G

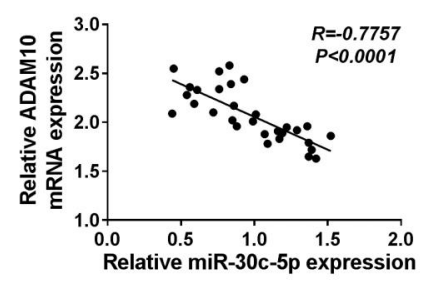

J

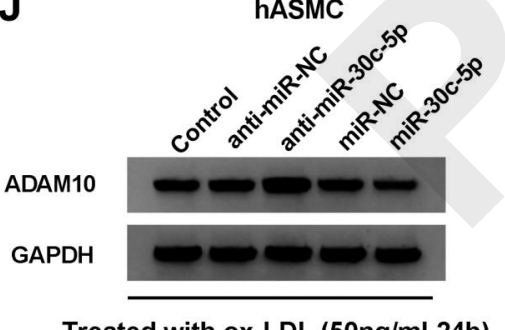

Treated with ox-LDL (50ng/ml,24h) | || || |

B

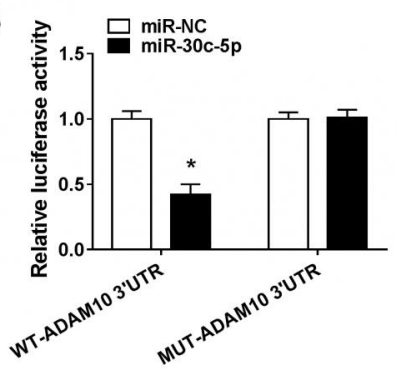

D
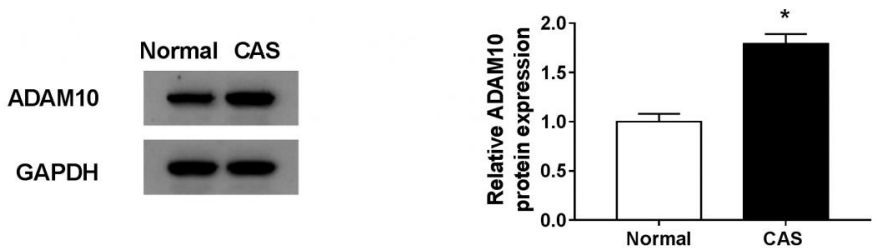

F

ox-LDL

$\begin{array}{llllll}(\mu \mathrm{g} / \mathrm{ml}) & 0 & 25 & 50 & 75 & 100\end{array}$ ADAM10

GAPDH
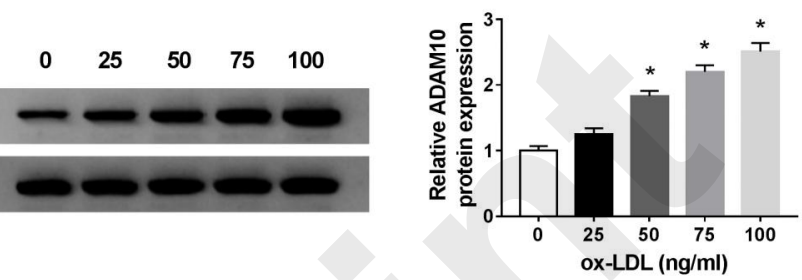

H

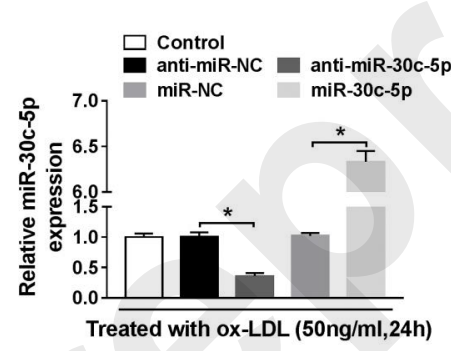

I

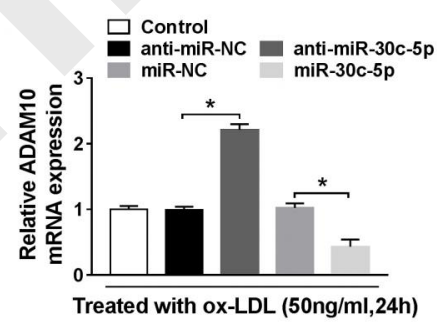

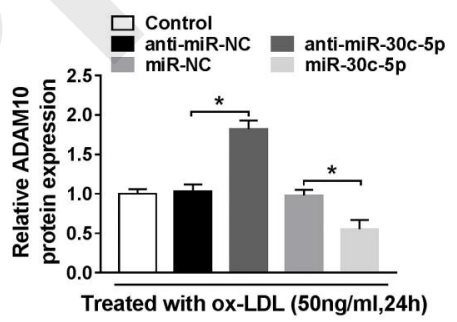


A

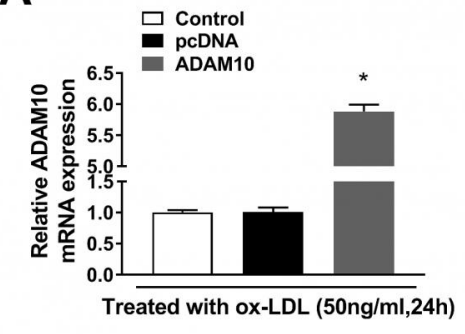

C

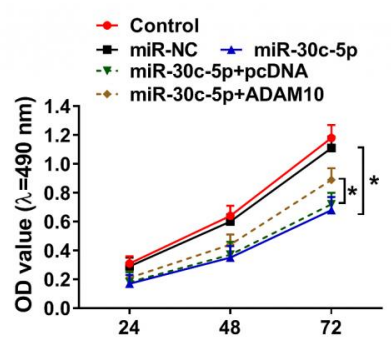

B

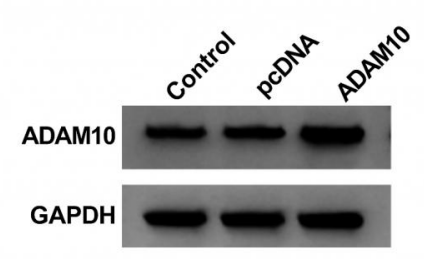

D

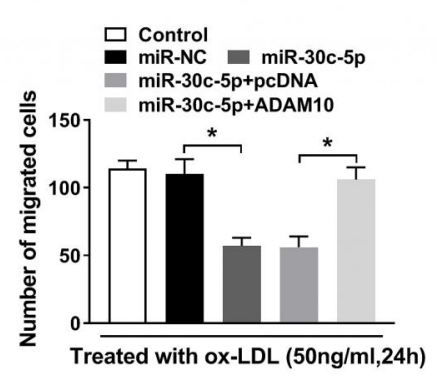

E

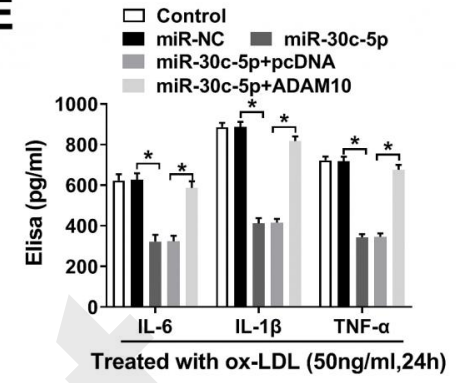

F

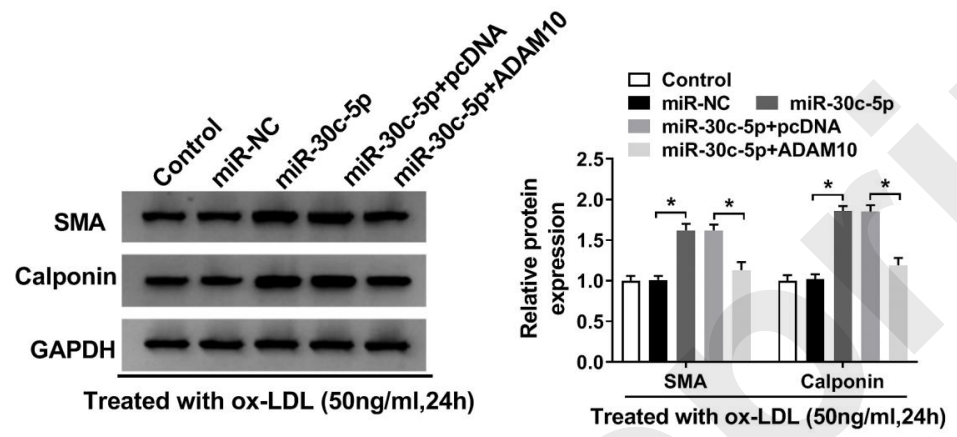




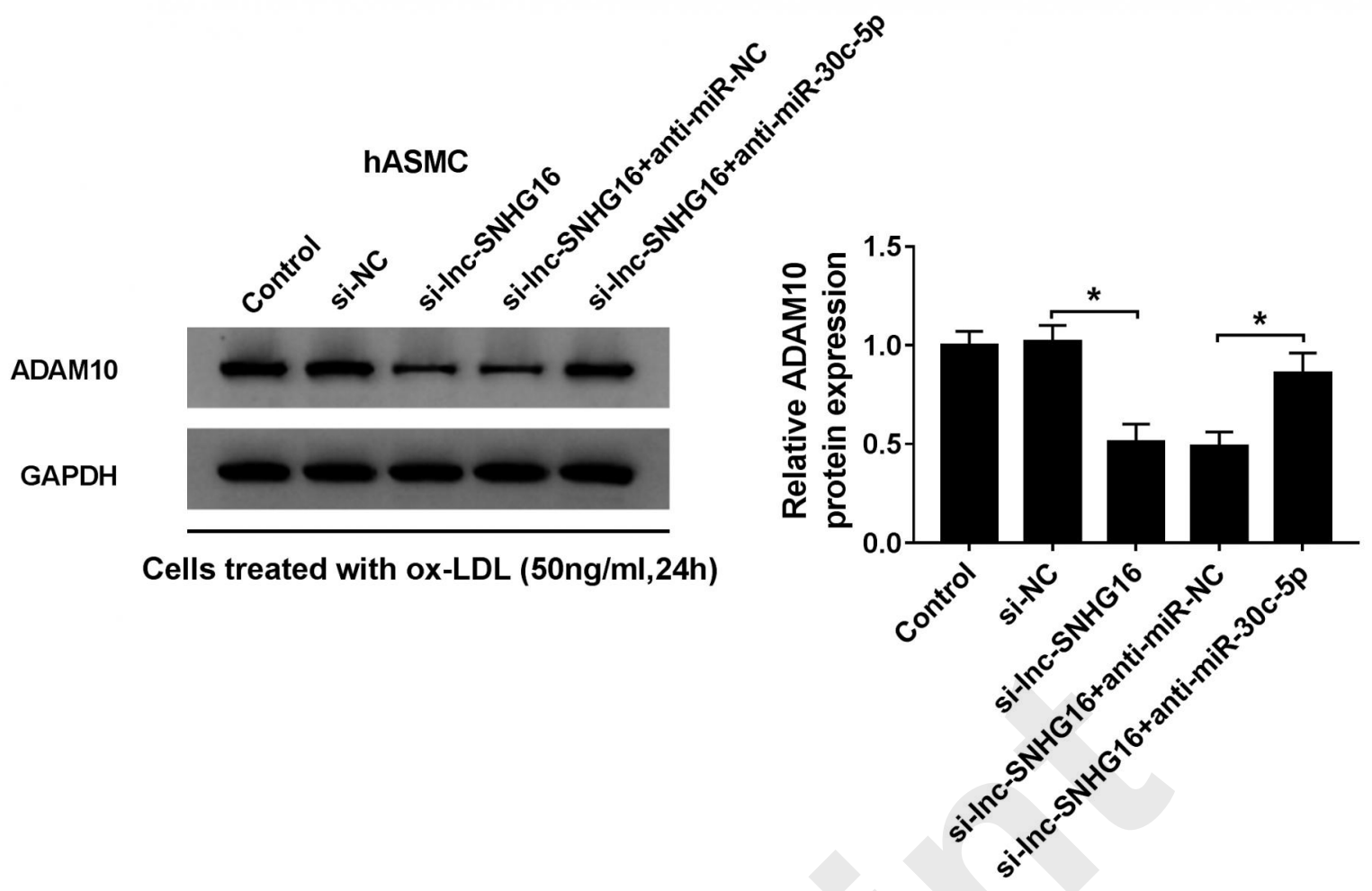

